\title{
Taking Care of Our Elders Through Augmented Spaces
}

\author{
Edgardo Avilés-López, Ismael Villanueva-Miranda, \\ J. Antonio García-Macías \\ Computer Science Department \\ CICESE Research Center \\ Km. 107 Carretera Tijuana-Ensenada \\ Ensenada, Baja California, México \\ \{avilesl,ivillanu,jagm\}@cicese.mx
}

\author{
Luis E. Palafox-Maestre \\ Facultad de Ciencias Químicas e Ingeniería \\ Universidad Autónoma de Baja California \\ Calzada Universidad \#14418, Mesa de Otay \\ Tijuana, Baja California, México \\ lepalafox@uabc.mx
}

\begin{abstract}
As the population ages worldwide, more and more efforts are being made toward providing the best conditions possible for our elders. In these efforts, technology plays a very prominent role. We present our proposal for ambient-assisted living spaces where an underlying sensorial infrastructure, coupled with sentient visors, can aid individuals in many daily tasks that promote a safer and healthier lifestyle. We also discuss some scenarios based on our contributions that serves as a showcase of what can be accomplished.
\end{abstract}

Keywords-ambient assisted living; sentient visors; ubiquitous computing

\section{INTRODUCTION}

In the course of the next years, worldwide population will constantly increase its average life expectancy. We can expect elderly forming a large part of the population as we can witness in some countries. Elders have special needs given the fact that the aging process will come with a considerable decline in capabilities making them prone to falls, forget things, and others. As technology advances so do the opportunities to come up with new tools that can drastically improve the lifestyle of our elders. Along with the particular requirements in assisting the elders, we must think in how they will interact with the tools and how to take advantage of the solutions available today so our contributions can be applied in the short term.

We start this article by discussing ambient assisted living; its scope, benefits and applications. Then, in Section 3 we introduce sentient visors, a technology designed to interact with services and applications in simple and easy means; in particular, its approach to elder users. In Section 4 we talk about our sensorial infrastructure for helping the elders. Later in Section 5, we exemplify those concepts through some scenarios in which we are currently working. Finally we present some conclusions and future work.

\section{Ambient Assisted Living}

Ambient Assisted Living (AAL) is a research area that has been gaining importance recently. Its main goal is to apply ambient technology to assist individuals in performing everyday activities. The social impact of AAL grows even

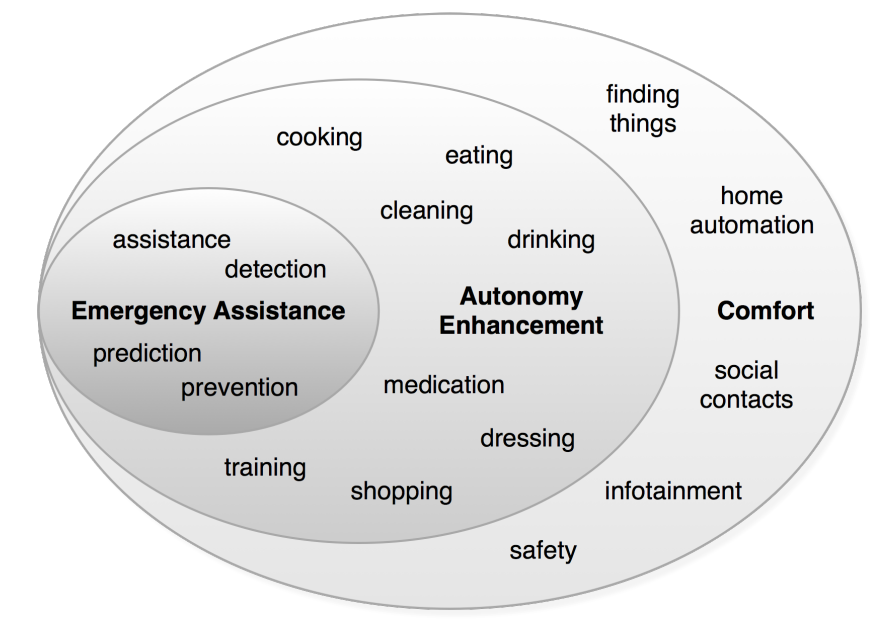

Figure 1. Homecare system domain.

more if it is applied on scenarios that involve physically impaired individuals and new alternatives on helping them to maintain certain degree of independence [1].

This technology opens the possibility to implement applications that offer specialized services according to specific application scenarios such as: assistance for daily activities, health monitoring, emergency systems and many others. According to Oppermann [2], AAL represent a new generation of systems that must comply with the following requirements:

- Invisible (embedded in clothing, watches, eyeglasses)

- Mobile (ability to be transported by the user)

- Spontaneous (ability to communicate dynamically with many nodes)

- Heterogeneous (the system must integrate several different types of nodes)

- Context-aware (ability to interpret user actions)

- Proactive (ability to infer behavior according to the activities of the user)

- Natural communication with the user, such as voice and gestures. 
- Adaptive (ability to react to uncommon situations that may occur)

In Figure 1, we show the three types of assistance in the homecare system domain introduced by Kleinberger [1], each of which presented as a subset: emergency assistance, autonomy enhancement, and comfort.

AAL systems development has focused on improving the quality of life on individuals with health problems and/or elderly people. In medical environments, AAL systems can provide assistance to patients with different kinds of cognitive impairments such as Alzheimer's or Parkinson's disease.

In elderly individuals, improving life quality through AAL environment is a goal that has been pursued recently [3]. This goal has gained greater importance because of the worldwide increase in average life expectancy [4]. For this reason, the assistance of elderly individuals for activities of daily living (ADL) has become an important social problem that can be solved (presumably) through the use of technology-augmented spaces such as AAL systems.

AlarmNET is an AAL system designed to provide assistance through a smart healthcare environment. It specifically targets assisted-living residents and others whom may benefit from continuous remote healthcare monitoring [5]. AlarmNET's network manages an audit trail and continuous medical history while preserving resident's autonomy, comfort and privacy. Thus, improving security and quality of life. The AlarmNET system [6] has been implemented as a network of MicaZ sensor nodes, Stargate gateways, iPAQ PDAs and PCs. Customized infrared motion, temperature, light, pulse and blood oxygenation sensors were used. Software components include: a TinyOS query processor and security modules for the motes; AlarmGate, an embedded Java application for power management, privacy, security, queries and client connections; Java resident monitoring and sensor data applications for PDAs and PCs; and a circadian activity rhythm analysis program.

\section{SENTIENT VisORS}

To further accomplish ambient assisted living we need to integrate many different devices into the environments of the users. But with this seamless integration of devices a new challenge of human-computer interaction arises. This is, how can users interact with devices that are "invisible" to them? And they are not just physically invisible but cognitively invisible as well. We also need to consider the scenario in which users of an augmented environment need to know what augmented physical objects are available for them to use, where they are, and once they encounter one of those, how to communicate with them. The interaction problem is further complicated if we talk about users of advanced age, as they commonly present difficulties for interacting with traditional software applications; so the migration from

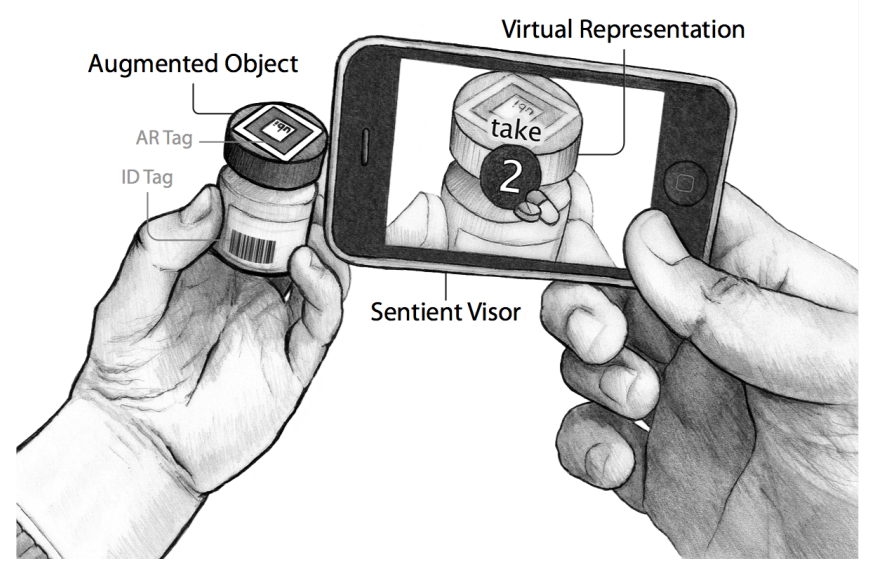

Figure 2. Scenario of use and anatomy of a sentient visor.

an already known paradigm of explicit interfaces to interaction with augmented objects may not be trivial. Elders need a clear and simple interaction mechanism that, taking in account their personal profile, presents them with the information they need when, where, and straightforwardly as they need it.

For instance, an elder person with diabetes may need to know the sugar contents of a particular food. For this user, the vitamins, minerals, fat, and other food elements are not as important as the sugar is. Even more, it would be useful to have a mechanism that allows the environment to know the personal dietary history and profile of the user, so it can present her with the digested food characteristics, an ingestion recommendation according to the diet indications given by his physician and stored in her profile.

Ambient displays are based on the idea of augmented environments and continuously present information in a very natural and simple way [7]. Those devices show information by physical means such as lights, sounds, images, and others. Users just need to have a glance or listen to them to be notified of an important incoming message or other information, so the interaction is very simple and straightforward. There is no need to push a button, select a menu, enter a command, nor any of the mechanisms present in traditional user interfaces, so they are very appropriate for elders.

Following the idea introduced by ambient devices, in our research group we have been working in what we call sentient visors. Sentient visors are user-carried devices that have a screen and a camera (so for instance, a tablet computer or a smartphone could be currently used as a sentient visor [8]). Those devices enable the interaction with augmented objects; to start an interaction the user just has to point the sentient visor to the object and the actual interaction takes place through an augmented reality scenario rendered on top of the video feed captured by the camera.

In Fig. 2 we can see the anatomy and scenario of use 
of the sentient visor concept for our previous example. To augment a physical object (in this case a package of food), we just need to attach an automatic identification tag (such as bar codes, RFID, or others) and an augmented reality tag, so there is no need for complex modifications on the subjects to be digitally enabled. Once the visor identifies the object been pointed at, it asks the environment for the scene to draw which will provide the needed interaction. Finally, the user is informed of the food characteristics through a graph and other indicators in 3D drawn over the video in the sentient visor. As the visor is not tied to the application and asks for what to draw, it can be extended to fit many different situations and application needs.

\section{Augmented Spaces for Helping the Elder}

There are two main technical components in our proposal: a sensorial infrastructure and sentient visors. In the following subsections we will detail how they work, and then in section $\mathrm{V}$ we will show through scenarios how they can be integrated to help in activities of daily life.

\section{A. Sensorial infrastructure}

Even when living at a carefully designed elderly-safe home, our elders are always at risk and accident-prone due to their ever decreasing abilities. We think that several technological elements can help reduce these risks and avoid accidents, or if accidents happen, caretakers can have a faster response. We have designed and implemented a platform based on the architecture shown in Figure 3, including modules to support the following functionalities: indoor location, video monitoring, behavior analysis, fall detection, voice commands, and decision-taking. We will now give a description of each of these modules.

The indoor location module estimates the user's position based on the signal's intensity of surrounding Wi-Fi access points. Based on the received signal, a neural network performs location estimation. The neural network has to be trained before the location service can be used, training is done by measuring Wi-Fi signal strengths at different points in the house and associating the measured signal strength levels with a specific location. The service has a function that enables the neural network to be trained by receiving three inputs: data (MAC address, signal strength), latitude and longitude. In our implementation, we took over 4,300 readings across 50 different locations using a laptop HP DV4000 as a client with an Intel IPW2200 wireless network card. On the client-side, we installed a Java application to send the information to the server. This information includes a dataset consisting of the MAC address and Wi-Fi signal strength (i.e., $\left.00: 13: 46: f_{2}: f_{C}: b 9,-67\right)$, latitude and longitude. Then using a k-means method, the readings were clustered to have a more localized reading. Once we had the clusters, these were used as input to a neural network. The goal we pursued when we did this was to increase

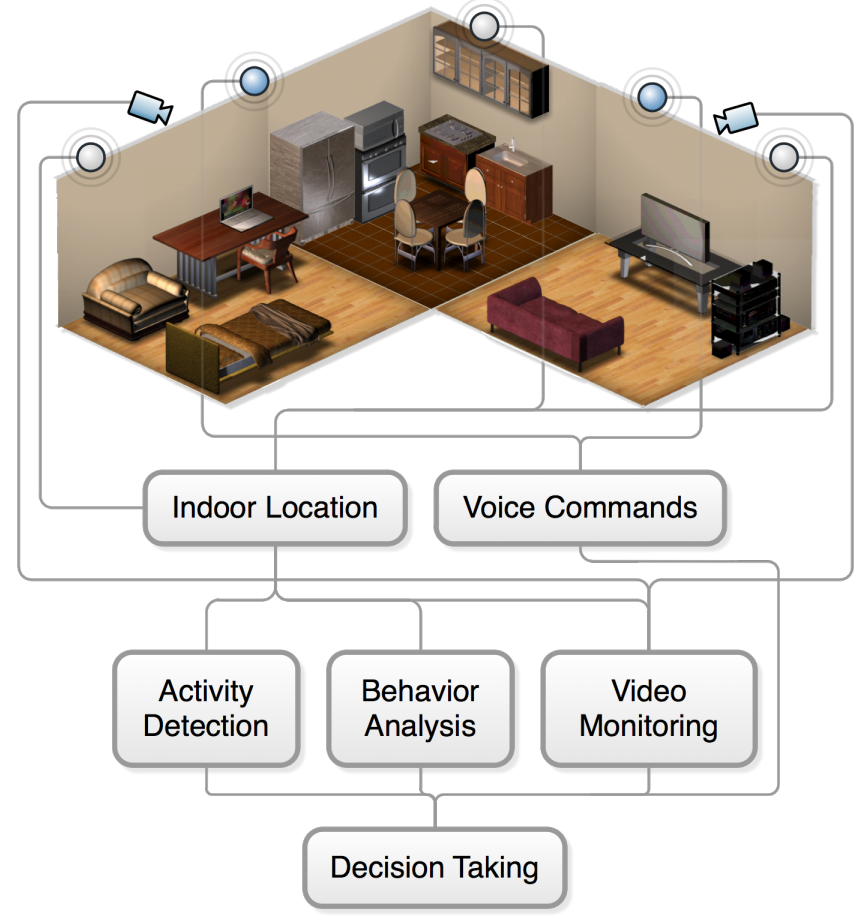

Figure 3. Our sensorial infrastructure.

the precision and reduce the processing time of location estimation.

Voice commands provide a very natural way of interaction where user effort is minimized. In our platform, we have implemented a subsystem where a sensor network captures sound, determines if it is within the human voice range, and if it is then forwards it to a base station (a full-fledged computer) to analyze it and determine if it is a command. For the implementation of this subsystem, we have installed a wireless network of small and cheap nodes with sound sensors. Reliability is an important factor, as it is important not to miss a command issued by the user, so we have deployed redundant nodes where sensing areas overlap; we made this decision based on the facts that nodes are failure-prone and have a low price. By doing this, if a node randomly fails we have other ones that could perform its capture and transmit commands without disrupting overall system functionality, thus assuring reliable voice capture. In a previous article we gave more in-depth details about the implementation and experimental results of using this voice command subsystem [9], so we will not repeat them here for the sake of brevity.

The activity detection module offers the possibility to infer certain low-level activities through the use of data provided by an accelerometer sensor. The activities are categorized as follows:

- standing up (from a seated position)

- seating down (from a standing up position) 
- walking

- climbing steps

- laying down (from a seated position)

- seating down (from a laying down position)

- falling down

Activity detection is a very crucial task for overall system functionality, it actually starts most of the higher level services provided. For instance, if certain activity is detected within a predetermined danger zone, this module sends a signal to the decision-taking module (which we will describe later) in order to take an appropriate action.

Given the fact that during the aging process, there is a considerable decline in motor capabilities, older adults become more prone to falls. Thus, it is important to note that fall detection is a very important function that is performed in the activity detection module that we mentioned earlier. Falls can occur in a variety of ways, whichever the case may be, this module must send a warning signal to the video monitoring and to the decision-taking module.

The video monitoring system serves as an auxiliary method for other systems. For instance, if the fall detection system indicates that there is a high probability that the user has fallen, an alert is issued to the caretaker and a video feed is activated, so it can be corroborated if the fall effectively took place or if it was a false alarm.

Dependent on the other modules, a behavior analysis module makes inferences, takes proactive actions, and issues alarms regarding the behavior of the users. Behavioral analysis includes determining patterns of conducts to facilitate proactivity. For instance, suppose that a person wakes up daily at about 7 AM, issues a voice command to open the curtains, proceeds to the bathroom and issues a command to have running water for taking a shower, and stays for roughly 30 minutes in the shower; then all these series of daily recurrent events can serve to indicate the system to open the curtains and have the water running when it is appropriate (also, taking into account location and movement). Also, if something deviates from the expected behavior, e.g., it is already 8:30 PM and the person is still in bed, then an alarm is issued and the caretaker is informed to take the appropriate actions.

The decision-taking module is arguably the most important one; this is because this module is in charge of taking specific actions that support target users. The decision is taken considering input signals provided by other modules. Once the decision has been taken, this module sends the appropriate signal to a specific actuator in order to perform a specific task, i.e. opening a faucet, making a phone call, sending a SMS message, activating an alarm.

\section{SCENARIOS}

We will now present some scenarios that illustrate how our platform can aid in many situations that can promote safer and healthier lifestyles for our elders.

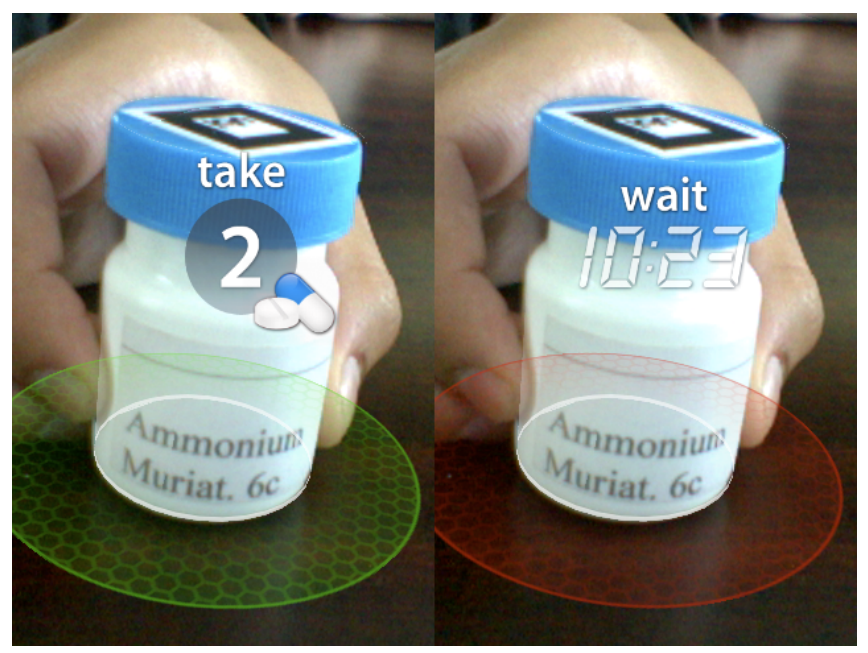

Figure 4. An aid for taking medications.

\section{A. Taking medications}

People who need to take many medications often struggle with remembering schedules and amounts of medication to take. Sometimes a medication is not taken simply because the person did not remember to do it, other times a medication is taken more than once, or not in the right amount. All these situations can lead to adverse, sometimes very serious consequences. In fact, polypharmacy is known to cause many problems worldwide [10].

Mr. Favela is a typical senior of advanced age who needs to take many medications daily. However, he often forgets to take these medications, sometimes he forgets which ones he already took and takes the same one twice or thrice, etc. Being concerned with this situation, his son living abroad gave him a cell phone that not only allows him to have conversations more often with his father, but also has installed sentient visor software for helping with medication intake. Now, when in doubt of which medication to take, Mr. Favela just needs to point at the bottle to see if it is the right one: the right bottle will show a green glow and the amount to take, while a wrong bottle will show a red glow and a clock counting backwards to the time for the next dose, as depicted in Figure 4.

The software also features audible alarms, so when Mr. Favela hears an event, he can point his cell phone at the bottles. Mr. Favela's physician can remotely use the same software to change the dosis by altering the number of pills to be taken or by setting a different schedule.

The medicine bottles have two important elements: an RFID tag to help retrieving the schedule and dosage information, and a label on top of the cap for orientation and helping in drawing the glow and other visual information presented in the display. 


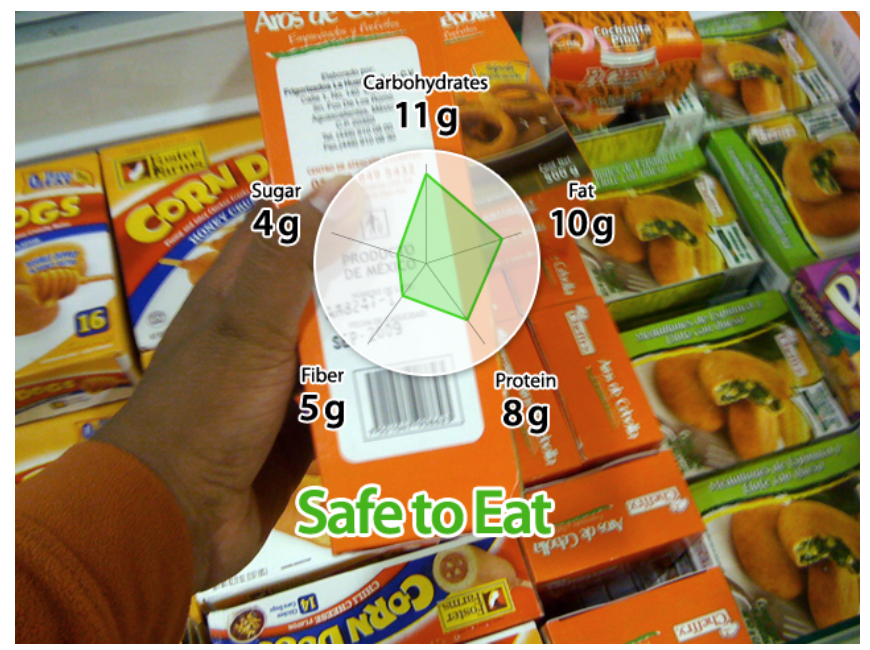

Figure 5. Dietary care showing the nutritional values and recommendation.

\section{B. Dietary care}

Due to her diabetic condition, Mrs. Tentori needs to be very careful with her diet. With this in mind, she downloaded a system for her iPod Touch that allows her to see the nutritional contents of products she is about to buy or eat.

When she is at the supermarket, she points at cans to see graphic charts indicating the nutritional values (e.g., carbohydrates, fat, sugar, etc.) of the products, which can allow her to make smarter buying decisions. Since she has slight visual impairments, it is always difficult for her to read directly at the small letters of the labels in the cans; so big and colorful charts provide her with better at-a-glance information (see Figure 5). Later during the day, she meets some friends for dinner at a restaurant. When she's presented the menu, she points at it with her iPod to get an augmented view where, as she picks some items, a table adds the amount of calories and sugar contents of her selection; if some value goes above her allowed daily intake, a visual alarm is presented. That way she knows what items she can take from the menu without incurring dietary problems. All this data is always remotely available so if her physician needs to know further about the history of the food she ingested he just needs to open his Web browser.

\section{Indoors guide}

During her frequent visits to health institutions, Mrs. Martinez gets disoriented and needs assistance in finding the doctor's office, the in-hospital pharmacy, etc. Luckily for her, many of the public institutions she goes to have augmented systems to help her.

Her doctor sent her to a clinic for specialized studies regarding a kidney condition she has. Once at the clinic she sees a "welcome" sign at the entrance (which has been augmented with a special label) and points at it with her cell phone. Once her location has been determined, a window is

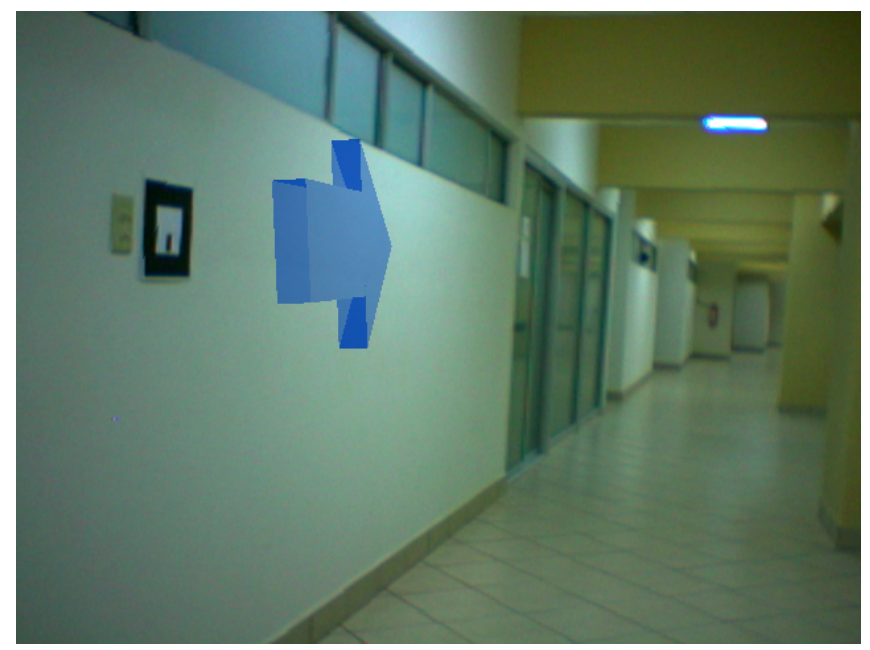

Figure 6. Interactive indoors guide.

displayed asking if she allows the ambient system to retrieve selected information from her medical record, she consents and she is instructed to point at the aisle to her left, where she will be directed to Dr. Moran's office. She then sees an arrow (similar to that in Figure 6) drawn on top of the real-time video feed she is getting from the phone's camera, and as she follows the arrow she gets to the right office.

If there is a problem, and Mrs. Martinez suffers a fall, the software is immediately aware of this and sends an alert to the hospital crew with the location and estimated urgency of the accident.

\section{Physician's aid}

During her morning rounds visiting patients at the hospital, Dr. Lugo always carries with her a tablet-sized computer. With this device, once she stands in front of the patient's bed she is able to view, overlayed on top of the live video feed, different types of charts and graphs indicating the patient's body temperature, heart rate, blood pressure, as well as info related to the treatment he is receiving.

\section{Vi. Conclusions and Perspectives}

Parting from the ambient assisted living concept, we have strived towards an augmented space that combines a sensorial infrastructure and sentient visors. We believe our proposals will contribute in improving the lifestyle of the elderly. At the moment, we are developing proof of concepts based on the scenarios discussed in this article using a working prototype of our platform. We are also exploring new scenarios and applications. We plan to conduct adoption and usability tests with a group of elder users to obtain feedback on how effective and appropriate are our contributions.

Although there are many useful technologies available to support an augmented space (e.g., WSN, RFID, AR, and others), there is a need for mechanisms to better detect and 
communicate the activity of the users. By instance, in our medications scenario, we would highly increase the value of our proposal by knowing if the user took or not the pills the application said him. In this way, we could alert his family and physician of his behaviour, or we could auto-request the pharmacy for medication resupply.

\section{ACKNOWLEDGMENT}

The work presented in this paper was funded with grants from CONACyT, the Mexican Council for Science and Technology.

\section{REFERENCES}

[1] T. Kleinberger, M. Becker, E. Ras, A. Holzinger, and P. Müller, "Ambient intelligence in assisted living: Enable elderly people to handle future interfaces," in Universal Access in Human-Computer Interaction. Ambient Interaction, 2007, pp. 103-112.

[2] R. Oppermann, R. Rashev, and K. Kinshuk, "Adaptability and adaptivity in learning systems," 1997.

[3] D. Hanak, G. Szijarto, and B. Takacs, "A mobile approach to ambient assisted living," in Proc. IADIS Wireless Applications and Computing, Lisbon, Portugal, 2007.

[4] United Nations. (2009) World population prospects the 2008 revisions. [Online]. Available: http://www.un.org/esa/population/publications/wpp2008/wpp2008_highlights.pdf

[5] G. Virone, A. Wood, L. Selavo, Q. Cao, L. Fang, T. Doan, Z. He, R. Stoleru, S. Lin, and J. A. Stankovic, "An assisted living oriented information system based on a residential wireless sensor network," in Proc. 1st Transdisciplinary Conference on Distributed Diagnosis and Home Healthcare (D2H2 2006), Arlington, VA, USA, 2006, pp. 95-100.

[6] A. Wood, G. Virone, T. Doan, Q. Cao, L. Selavo, Y. Wu, L. Fang, Z. He, S. Lin, and J. Stankovic, "ALARM-NET: Wireless sensor networks for assisted-living and residential monitoring," Department of Computer Science, University of Virginia, Tech. Rep. CS-2006-11, 2006.

[7] J. Favela, M. Tentori, D. Segura, and G. Berzunza, "Adaptive awareness of hospital patient information through multiple sentient displays," International Journal of Ambient Computing and Intelligence, vol. 1, no. 1, pp. 27-38, 2009.

[8] A. Wright, "Get smart," Communications of the ACM, vol. 52, no. 1, pp. 15-16, 2009.

[9] L. E. Palafox-Maestre and J. A. García-Macías, "Wireless sensor networks for voice recollection in ubiquitous home environments," in International Symposium on Wireless and Pervasive Computing (ISWPC 2009), Melbourne, Australia, Feb. 2009.

[10] L. Baro-Manuela, "Deterioro de la movilidad fisica y continuidad de cuidados," in Comisión para el desarrollo de la enfermería en el servicio andaluz de salud (CODAE), Apr. 2002, vol. 3, no. 2 . 